\title{
Innovative Technique of Primary Percutaneous Coronary Intervention in Patients with Impaired Renal Function using Ultra Low Volume Contrast Agent Using Side Branch Wire as a Marker
}

\author{
Santosh Kumar Sinha, FACC, FAESC, FSCAI* (D), Awadesh Kumar Sharma (D, \\ Mahmodullah Razi (D) and Umeshwar Pandey
}

Department of Cardiology, LPS Institute of Cardiology, GSVM Medical College, India

*Corresponding author: Santosh Kumar Sinha, FACC, FAESC, FSCAI, Associate Professor, Department of Cardiology, LPS Institute of Cardiology, GSVM Medical College, Kanpur, Uttar Pradesh, 208002, India, Tel: +91-9670220088, Fax: +910512-2556199, 2556521

\begin{abstract}
Contrast-induced nephropathy (CIN) is a serious complication in patients with chronic kidney disease with coronary artery disease who undergo percutaneous coronary intervention $(\mathrm{PCl})$, and is associated with higher morbidity and mortality in short and long term. The limiting volume of contrast agent is safest and most reliable strategy to prevent $\mathrm{CIN}$. Here, we report a case of suc $\urcorner$ cessful primary $\mathrm{PCI}$ using only $13 \mathrm{ml}$ of contrast (iodixanol) of mid left anterior descending artery where wire in diagonal branch served as a landmark in a 75-year-old diabetic and hypertensive male who had presented with ST segment elevation anterior wall myocardial infarction and had marked renal dysfunction having serum creatinine of $3.3 \mathrm{mg} \%$. There was no change in basal renal function after the procedure and safely discharged.
\end{abstract}

\section{Keywords}

Contrast-induced nephropathy, Percutaneous coronary intervention, Acute coronary syndrome, Contrast agent, Left anterior descending artery

\section{Introduction}

Contrast-induced nephropathy (CIN) is associated with increased morbidity and mortality including the need for renal replacement therapy (RRT) which is defined as absolute $(\geq 0.5 \mathrm{mg} / \mathrm{dL})$ or relative increase $(\geq 25 \%)$ of serum creatinine from baseline after $48-72$ hours following contrast administration, when alterative explanations have been excluded [1,2]. Peri-procedural hydration and minimum possible volume of contrast are the only established measures to prevent CIN $[1,3]$. The risk of $\mathrm{CIN}$ which is $0.6-2.3 \%$ in general population and approaching to $20 \%$ in high risk patients, and requirement of RRT are the prime factors responsible for underutilization of percutaneous coronary intervention $(\mathrm{PCl})$ in patients with established chronic kidney disease or those with baseline renal impairment [4].

\section{Case Report}

A 75-year-old diabetic and hypertensive male presented with chest heaviness with sweating of 6 hours duration. An electrocardiogram showed marked ST elevation in $\mathrm{V}_{1}-\mathrm{V}_{6}$. Routine haemogram and biochemistry was normal except deranged renal function with serum creatinine of $3.3 \mathrm{mg} \%$. His body weight was $68 \mathrm{~kg}$. Echocardiography revealed mild concentric left ventricular hypertrophy, regional wall motion defect in left anterior descending territory (LAD), and impaired systolic function with an ejection fraction of $40 \%$. His coronary angiogram was done through transfemoral approach after proper consent revealing critical lesion with $90 \%$ stenosis in mid left anterior descending (LAD) artery just after large diagonal branch, normal left circumflex

Citation: Sinha SK, Sharma AK, Razi M, Pandeyv U (2021) Innovative Technique of Primary Percutaneous Coronary Intervention in Patients with Impaired Renal Function using Ultra Low Volume Contrast Agent Using Side Branch Wire as a Marker. Int J Clin Cardiol 8:222. doi.org/10.23937/23782951/1410222

Accepted: March 20, 2021: Published: March 22, 2021

Copyright: (c) 2021 Sinha SK, et al. This is an open-access article distributed under the terms of the Creative Commons Attribution License, which permits unrestricted use, distribution, and reproduction in any medium, provided the original author and source are credited. 


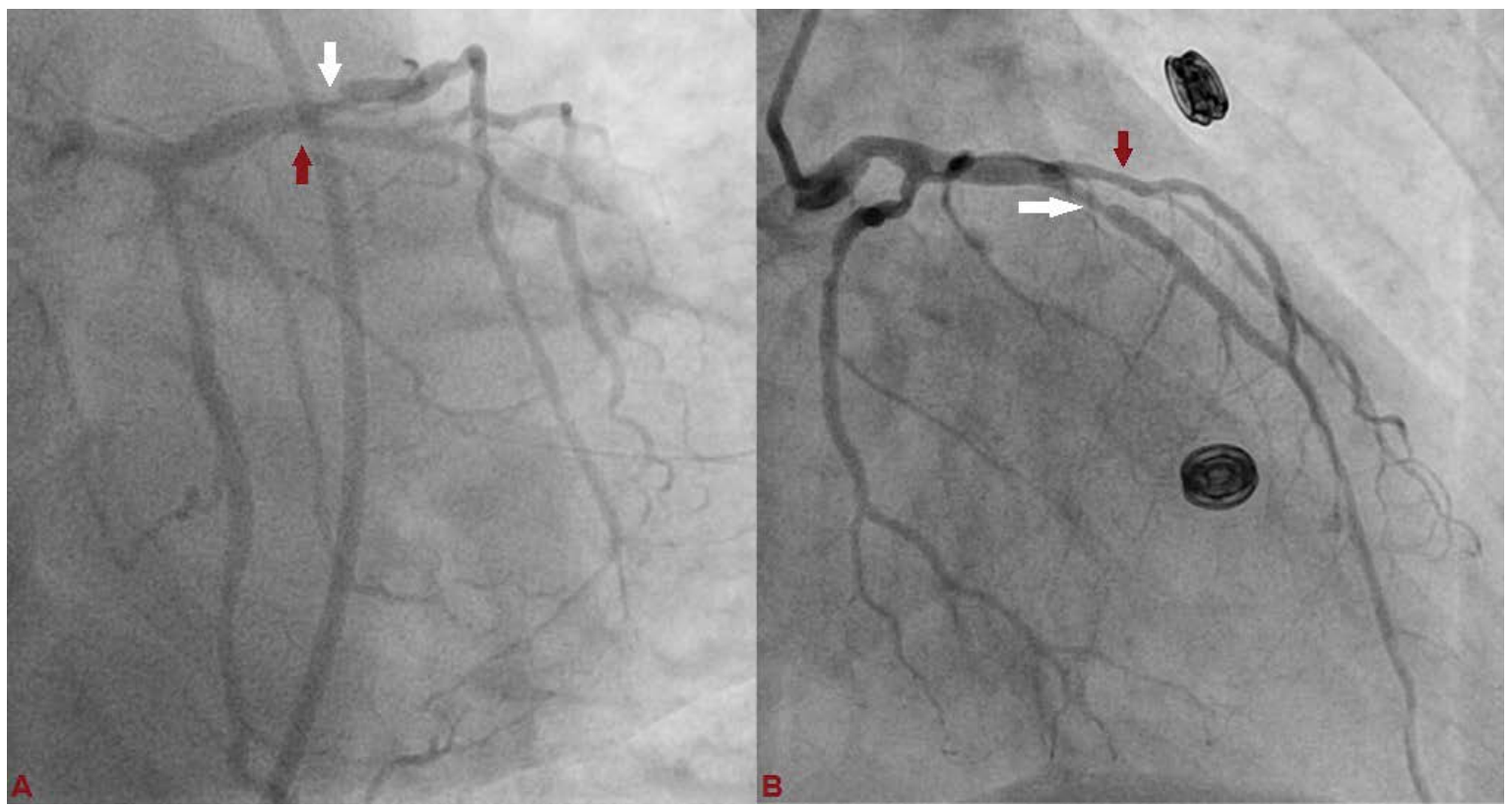

Figure 1: Left anterior descending (LAD) artery revealing critical lesion with $90 \%$ stenosis in mid just after large diagonal branch (D1), and normal left circumflex artery. (A: Antero-posterior view with caudal angulation; B: Antero-posterior view with cranial angulation).



Figure 2: Left anterior oblique view showing normal flowing right coronary artery.

territory, and right coronary artery (Figure 1 and Figure 2). The coronary angiogram was completed using $9 \mathrm{ml}$ of contrast. The contrast used was iodixanol which is an iso-osmolar contrast agent. Primary $\mathrm{PCl}$ was planned subsequently. Intravenous heparin (100 U/Kg) was administered. Based on all the baseline factors, it was a high risk $\mathrm{PCl}$ carrying a profound risk of $\mathrm{CIN}$. To minimize the risk of $\mathrm{CIN}$, intravenous normal saline infusion was started. Prior to $\mathrm{PCl}$, initial angiogram with same angle of projection was uploaded to the monitor for $\mathrm{PCl}$ guid- ance. Left main artery was engaged with $6 \mathrm{~F}$ extra backup guiding catheter (EBU; Medtronic, USA) which was confirmed by entry of the runthrough wire (Terumo, Japan) into the coronary artery. An exaggerated curve was created to facilitate the wiring of LAD. Additional runthrough wire was placed in first diagonal branch (D1) based on the previous angiogram (Figure 3). The side branch wire served as an important landmark to guide $\mathrm{PCl}$ and to protect the side branches as well. The balloon was positioned just after D1 which was used as a landmark for proximal end for stent placement. Lesion was gradually predilated with $2 \times 10$ and $2.5 \times 10$ pantera leo semi compliant balloons (Biotronik, Germany). $3 \times 23 \mathrm{~mm}$ Endeavour Resolute (Zotarolimus eluting stent; Medtronic, USA) stent was positioned across the lesion keeping the proximal end at the crossing of LAD and D1 wire which was serving as the origin of D1 (Figure 3). It was deployed at 13 atm pressure (Figure 4). As it was not fully expanded, it was serially post dilated by $3 \times 10$ and $3.5 \times 10$ non-compliant Minitrak balloon (Abott, USA) at 24 atm pressure. The stent apposition was checked under stent boost. After confirmation of stent optimization with stent boost, final angiography with $4 \mathrm{ml}$ contrast injection revealed successful results (Figure 5). Therefore, $\mathrm{PCl}$ was completed using $13 \mathrm{ml}$ of contrast. He was clinically stable for 2 days without CIN (Serum creatinine $3.5 \mathrm{mg} / \mathrm{dL}$ ). She was discharged 2 days after $\mathrm{PCl}$ with appropriate drugs and has been uneventful.

\section{Discussion}

CKD and CAD share several risk factors, and therefore patients with CKD have a higher atherosclerotic burden [5]. Presence of advanced atherosclerosis is also major risk factors for $\mathrm{CIN}$, thus complicating $\mathrm{PCl}$ in pa- 


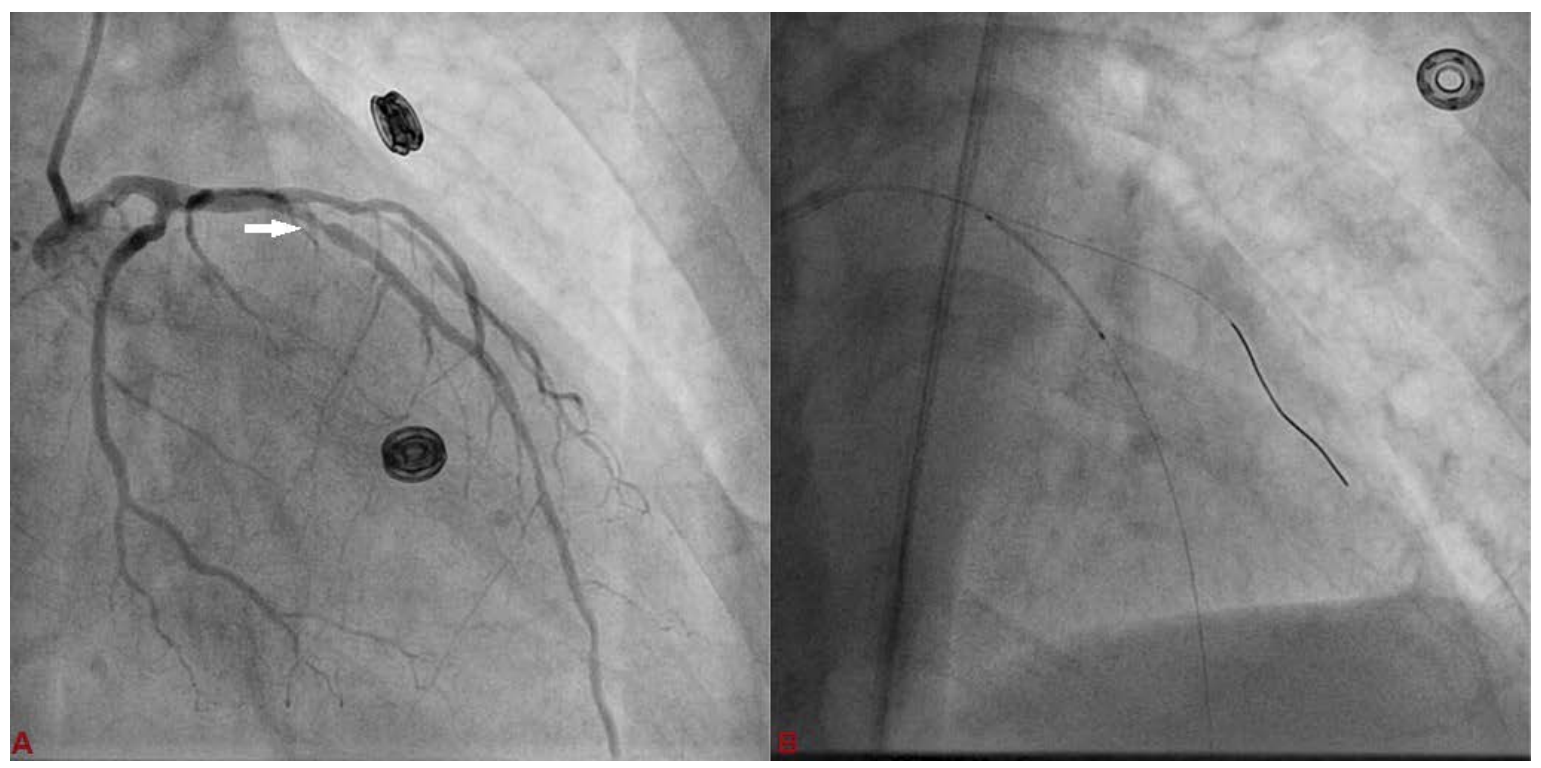

Figure 3: A) Basal angiographic view of left system used as anatomical reference to mark position of $L A D$ and $D 1$; $B$ ) $3 \times$ $23 \mathrm{~mm}$ Endeavour Resolute stent was positioned across the lesion keeping the proximal end at the crossing of LAD and D1 wires which was serving as the origin of D1.



Figure 4: Stent was deployed at 13 atm pressure.

tients with CKD and thereby making revascularization an under-utilized option in this subset of patients. These patients who undergo revascularization have survival advantage over medical therapy alone based on observational studies although randomized trials are lacking [5]. However, concern regarding $\mathrm{CIN}$ and requirement of RRT offsets the performance of revascularization in patients with CKD. Pre-existent renal disease is the strongest independent predictor for development of $\mathrm{CIN}$ and the requirement for RRT, which develop in 27 and $4 \%$ of patients with severe CKD, respectively. The 2014 European Society of Cardiology/European Association for Cardio-Thoracic Surgery (ESC/EACTS) guidelines on myocardial revascularization advocate ad hoc rather than staged $\mathrm{PCl}$ in patients with $\mathrm{CKD}$ and extensive

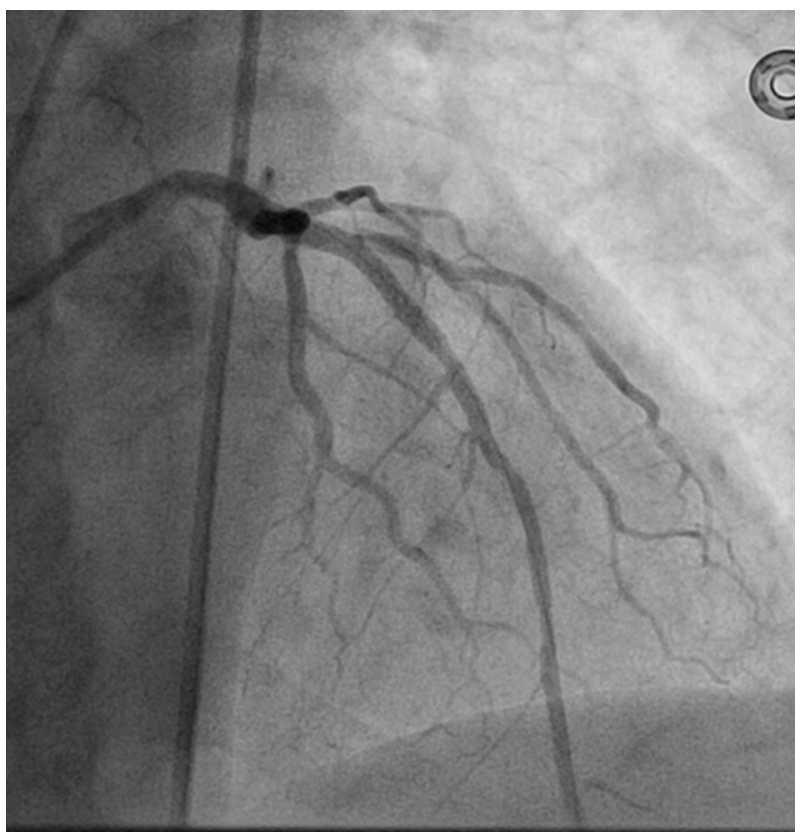

Figure 5: Final angiography with $4 \mathrm{ml}$ contrast injection revealed successful PCI of LAD with TIMI III flow.

atherosclerosis, if substantial contrast use in one single invasive procedure can be avoided, to minimize the risk of acute kidney injury secondary to atheroembolism [6]. Despite many proposed modality to treat $\mathrm{CIN}$, prevention is the best strategy to improve the outcome after $\mathrm{PCl}$ as in-hospital, 1-year, and 5-year mortality rate are significantly higher in patients who develops CIN compared to those who don't $(1.4 \%$ vs. $22 \%),(3.7 \%$ vs. $12.1 \%)$, and (14.5\% vs. $44.6 \%$ ) respectively [7]. Contrast volume as minimum as possible is the safest and most reliable strategy which can be done in several ways including pre-interventional imaging to plan procedure, images of target vessel anatomy as reference, use of diluted contrast, iso-osmolar contrast, imaging modal- 
ities such as intravascular ultrasound (IVUS), and hybrid imaging [7]. IVUS guided $\mathrm{PCI}$ has 3 -fold lower usage of contrast compared to angiography-only approach, thus making it an attractive alternative imaging tool to guide $\mathrm{PCl}$ [7]. The drawback is that it is costlier, requires operator expertise, a learning curve, and accurate image interpretation which make it potentially not very attractive. Furthermore fluoroscopy time, the number of cine runs, or radiation dose may be higher for less experienced operators though it was not reported in study by Mariani J Jr, et al. [8]. IVUS carries an advantage that it gives valuable information on cross-sectional anatomy, appropriate landing zone, diameter and length of stent, and accurate apposition which therefore minimizes the complications. Although in our case, lesion was type A, considering the baseline profile of the patient, risk of $\mathrm{CIN}$ was very high which made it an extremely high risk procedure. In our case, minimal dosage of contrast at the end of the procedure was used to confirm any injuries in the form of dissection, apposition, and status of side branch. Therefore, it was demonstrated that ultra-low contrast volume reduces the rate of $\mathrm{CIN}$ in patients with chronic renal disease who undergo $\mathrm{PCl}$, which can be done with limited resources where IVUS is not available. The lack of worsening renal function following $\mathrm{PCl}$ in our case suggests that $\mathrm{CIN}$ is the major cause of acute nephropathy following revascularization, and staged procedure may be considered as an alternative in extremely high-risk group of patients.

\section{Conflict of Interest}

None.

\section{Sources of Support (if applicable)}

Disclosure of funding received for this work from any of the following organizations: National Institutes of Health (NIH); Welcome Trust; and other(s) - None.

\section{Statement of Equal Authors' Contribution}

All authors made equal contribution.

\section{References}

1. Mehran R, Nikolsky E (2006) Contrast-induced nephropathy: Definition, epidemiology, and patients at risk. Kidney Int Suppl 100: S11-S15.

2. Dangas G, lakovou I, Nikolsky E, Aymong ED, Mintz GS, et al. (2005) Contrast-induced nephropathy after percutaneous coronary interventions in relation to chronic kidney disease and hemodynamic variables. Am J Cardiol 95: 1319.

3. Brar SS, Aharonian V, Mansukhani P, Moore N, Shen AY, et al. (2014) Haemodynamic-guided fluid administration for the prevention of contrast-induced acute kidney injury: The POSEIDON randomised controlled trial. Lancet 383: 18141823.

4. Nayak KR, Mehta HS, Price MJ, Russo RJ, Stinis CT, et al. (2010) A novel technique for ultra-low contrast administration during angiography or intervention. Catheter Cardiovasc Interv 75: 1076-1083.

5. Ali ZA, Karimi Galougahi K, Nazif T, Maehara A, Hardy MA, et al. (2016) Imaging- and physiology-guided percutaneous coronary intervention without contrast administration in advanced renal failure: A feasibility, safety, and outcome study. Eur Heart J 37: 3090-3095.

6. Windecker S, Kolh P, Alfonso F, Collet JP, Cremer J, et al. (2014) 2014 ESC/EACTS guidelines on myocardial revascularization: The Task Force on Myocardial Revascularization of the European Society of Cardiology (ESC) and the European Association for Cardio-Thoracic Surgery (EACTS). Developed with the special contribution of the European Association of Percutaneous Cardiovascular Interventions (EAPCI). Eur Heart J 35: 2541-2619.

7. Lee OH, Ahn CM, Kim JS, Kim BK, Ko YG, et al. (2017) Successful treatment of unprotected left main coronary bifurcation lesion using minimum contrast volume with intravascular ultrasound guidance. Yonsei Med J 58: 10661070.

8. Mariani J Jr, Guedes C, Soares P, Zalc S, Campos CM, et al. (2014) Intravascular ultrasound guidance to minimize the use of iodine contrast in percutaneous coronary intervention: The MOZART (minimizing contrast utilization with IVUS guidance in coronary angioplasty) randomized controlled trial. JACC Cardiovasc Interv 7: 1287-1293.

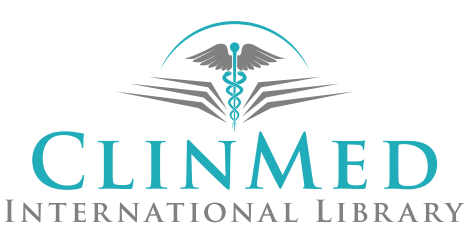

